\title{
Changes and Differences in Body Composition and Strength Abilities of Athletes in Fitness and Bodybuilding at Different Intervals of Rest
}

\author{
Jan Caha, Jan Cacek, Jan Ondráček,Tereza Králová, PřemysI Šimon, Simon Šiška, Klára Vomáčková, \\ Michal Žák, Tomáš Vodička
}

Masaryk University Brno, Faculty of Sports Studies

\begin{abstract}
Efforts to increase muscle mass (hypertrophy) are required in sports that are dedicated to lifting or weightlifting. Bodybuilding methods with moderate load and relatively short rest between sets and exercises for a selected muscle area, that induce a high amount of metabolic stress, can be effective for hypertrophy of muscle tissue.

The paper deals with changes in body composition (muscle mass, adipose tissue and bone mass), and changes in the manifestations of maximal strength of the extensors and flexors of the lower and upper limbs in relation to different periods of rest (30 s, 60 s, 120 s). The research was carried out on 23 participants, aged 18-30 years, who were elite, natural bodybuilders, during a 5-week intervention of bodybuilding training.

There are several outcomes which resulted from the study. At a statistical significance level of $5 \%$, there was no significant difference in muscle tissue hypertrophy between the groups. The athletes who had a 60 second ret period achieved a two-fold increase in the percentage of fat free mass compared to the other two groups. At a statistical significance level of 5\%, we noticed significant differences in the maximum force increase of the knee joint flexors, only in Group 3 (120 s) and by up to 11\%. Significant differences in muscle strength increase were also observed in groups 2 and 3 in the elbow extensors and in the elbow flexors of Group 1 only.
\end{abstract}

Key words: hypertrophy, muscle mass, body fat, biceps, quadriceps

\section{INTRODUCTION}

Efforts to increase muscle mass or hypertrophy are required and targeted in sports that are dedicated to lifting or weightlifting, in aesthetic sports like fitness and bodybuilding, and generally in sports that require performance based on highly developed strength capabilities. Consequently, finding the optimal way to build muscle mass is essential for bodybuilding and fitness. The primary goal of this study was to investigate this. (3)

Due to the strong correlation between muscle size and muscle strength, increasing muscle mass in athletes with a strong force element (powerlifting, weightlifting, rugby) is one of the main goals of their training. However, muscle mass is also the most crucial element in bodybuilding, fitness and all the disciplines of coordination-aesthetic sports included in these categories. Here, the athletes are judged by the amount and quality of their lean muscle mass. This can also be seen at the recreational level of fitness and bodybuilding, where there is a growing trend for individuals to attempt to increase or build muscle mass from an aesthetic and functional point of view. The amount of muscle mass and balance of individual sport is one of the most essential elements of these sports, and may even be essential for success in sporting competitions. $(3,7)$. 
Appropriately applied strength training leads to both muscle mass and strength development. The mechanism and impact of strength training can be summarized in 3 key points $(3,2,4,6)$.

\section{The mechanism of training leading to hypertrophy}

1. Mechanical tension - is associated with the use of training weights - the size (intensity) of training weights (load), which put the muscle under the maximum possible (new) tension, which subsequently induces muscle growth and recruitment of new muscle spindles = myofibrillar hypertrophy $(1,2,3)$.

2. Muscle damage - muscle damage and microtrauma formation stimulates the muscle to grow but at the same time it must not be high enough to exceed the regenerative capacity and muscle protein catabolism should not outweigh the production of new proteins. For greater muscle damage, it is advisable to put more emphasis on the eccentric phase of movement (control of the weight in eccentric phase), which can last for 3-4 seconds and is associated with higher formation of waste metabolites (metabolic stress) $(1,2,3)$.

3. Metabolic stress - a series with longer time under tension (TUT) puts considerable demands on the metabolism of muscle cells, particularly in larger muscle groups such as the legs or back, as well as the organism as a whole. In long-term series, the muscle moves into a state of oxygen deficiency (hypoxia). This condition causes the leaching of many substances that are expected to have a positive impact on muscle proteosynthesis = sarcoplasmic muscle hypertrophy $(1,2,3)$.

Strength training has several highly significant impacts on the body of a sports person. The most obvious impacts are related to the amount of muscle mass, which is represented by the muscle cross-section and muscle strength. Properly applied strength training can lead to a maximum strength increase of more than $20 \%$ in a 21 -week training program. A similar effect on muscle mass and strength has also been described in strength-training women $(9,8)$.

Muscle mass and its strength both decrease during ageing, especially from older adulthood, both in men and women (10). It is believed that the decrease in muscle mass is due to a reduction in the size of a number of different individual muscle fibers, especially muscle fibers with the fastest activation - fast-twich muscle fibers (11). The decrease in age-related strength may also be due to a reduction in the maximal voluntary activation of the agonist muscle or changes in co-activation of the antagonist working muscle. The decrease in muscle mass and strength associated with age is not surprising, as ageing is very often associated with a decrease in the number of daily activities, but also with a general decrease in the number of activities and their intensity and thus lower activation of individual muscle groups and fibers $(12,13,14)$.

Progressive strength training not only in young and middle-aged people but also in older men and women, can lead to a significant increase in strength and muscle mass. This could be primarily due to the observed significant nerve adaptations, especially during the first weeks of training $(12,13,27)$, suggesting a significant increase in maximal electromyographic (EMG) activity of trained agonist muscles associated with decreased antagonist coactivation. This has been particularly found in older women $(12,13,14)$.

Various types of strength training cause acute and in some cases, chronic hormonal changes, which appear to play an essential role in mediating hypertrophic signaling reactions (15). The three most studied of these hormones are insulin growth factor (IGF-1), testosterone and growth hormone (GH). We regard these as the most important in terms of anabolic reactions and responses to strength training, both in their acute and chronic response and changes concerning strength training (2).

As we have already described, the initiation of muscle hypertrophy by strength training seems to reflect three essential elements: mechanical tension, muscle damage and metabolic stress $(4,6)$, 
ultimately influenced by several training factors. Relevant variables which may have an influence on the outcome here, include: intensity, weight, rest, choice of training or exercises, muscle failure and repetition rate, i.e. different training methods or practices in general (2).

\section{Break between training series - actual data synthesis}

Rest periods between training sets significantly affect overall training time, acute regeneration of forces and energy substrates, and thus also impact on metabolic stress and the subsequent length of a work series, and the ability to deliver maximum power. These usually range from 30 to 300 seconds (s). They can be divided into short rest periods (up to $30 \mathrm{~s}$ ), medium rest periods (up to $60 \mathrm{~s}$ ) and long rest periods (180-300 s) (22).

Short rest pauses (up to $30 \mathrm{~s}$ ) generate high metabolic stress, but do not provide enough rest for subsequent maximum power. However, they are believed to be effective in building muscle mass $(24,26)$.

Moderate breaks are a compromise between short and long. They provide sufficient time to regenerate the energy reserves used in non-maximum workout training and cause high metabolic stress (23). They are also associated with higher hypoxia, which may subsequently have a more significant impact on muscle mass, but also with a higher hormonal "peak" in response to strength training (20).

Long rest pauses are advantageous for maximum regeneration of forces between series and thus possible maximization of mechanical tension and utilization of higher loads. Metabolic stress is not as high as for short or medium rest breaks $(21,25)$.

The findings of previous studies that have investigated which length of rest period are more beneficial in terms of more pronounced muscle hypertrophy, have varied. More often, experts tend to believe that more pronounced muscle hypertrophy is noticed at pauses longer than 60 seconds, as well as an increase in muscle strength (16). Rest pauses, which help to achieve better results in muscular hypertrophy of the lower body, could also be beneficial for muscle hypertrophy (17).

\section{Aims of the study}

The aim of this study is to determine which rest periods have a higher influence on muscle tissue growth, decrease the amount of fatty tissue, and also to decide which type of rest leads to higher strength gains in typical strength training, which may be referred to as "hypertrophy training for bodybuilders". Details on the training plan we used in the research can be found in supplement 1 .

\section{METHODS}

\section{Experimental Approach to the Problem}

Each subject visited the laboratory on 2 occasions separated by 5 weeks. On visit 1, the subjects were assessed for body composition (FFM - fat free mass, fat, muscle mass, bone density) and maximal strength of the arm extensors and flexors, and the leg extensors and flexors. On the second visit, the same values were examined as during the first visit, ie body composition on a DEXA device and the maximum strength of the extensors and flexors of the arms and legs on an isokinetic dynamometer.

\section{Subjects}

A group of 23 men aged 18-35 years ( $n=23$, with 7-9 in each group), who were performance level, natural bodybuilders, were selected for the study. All subjects had more than 5 years of 
training experience and were trained in proper exercise technique. All subjects had been free of any lower-body, musculoskeletal, or neuromuscular injuries for the previous 6 months. All subjects claimed no current or previous use of any anabolic steroids.

All subjects refrained from other physical activities that could have affected the outcome of the experiment. Simultaneously, they followed the prescribed nutritional plan, where they consumed protein at the level of $2 \mathrm{~g} / \mathrm{kg}$ body weight and did not use any specific food supplements, which may have had a significant impact on performance. All subjects were advised to maintain a balanced energy intake and typical nutrition comprised of a dietary intake of 40-50\% carbohydrates.

Before visiting the laboratory the subjects were instructed in further aspects of dietary behavior, including the omission of alcohol and stimulants, such as nicotine.

\section{Procedures}

\section{Resistance exercise protocol}

The training program was clearly explained to all subjects and any individual discrepancies in exercise technique were standardized. The training protocol was identical for all subjects (see supplement 1), except for the difference in rest time between training sets. The subjects followed the training protocol for 5 weeks.

\section{Bone densitometry machines DEXA (body composition)}

Dual energy X-ray absorption spectrometry (DEXA) has high accuracy for body composition analysis. It was used to determine body fat, net body weight, total body weight, bone density and adipose tissue distribution of all subjects.

\section{Isokinetic dynamometer (muscle strength)}

An isokinetic contraction is a muscular contraction that accompanies a constant velocity of limb movement around a joint. The velocity of movement may be maintained constantly by using a special dynamometer. The resistance of the dynamometer is equal to the muscular forces applied throughout the range of movement. This method allows the measurement of muscular forces under dynamic conditions and provides optimal loading of the muscles. However, during movements in the vertical plane, the torque registered by the dynamometer is the resultant torque produced by the muscular and gravitational forces (5).

\section{Statistical methods used in research}

Comparison of the FFM difference, fat and \% fat for the 3 groups according to the length of rest (30, 60 or $120 \mathrm{~s}$ ) was performed using an ANOVA. The presumption of data normality was verified by the Shapiro-Wilk test, the presumption of homogeneity of variance by the Leven test.

Descriptive statistics were calculated for each variable: the mean, standard deviation, median, minimum, and maximum, which are presented in both tables and box charts. The selected significance level was 0.05, calculations were performed using the program Statistica.

\section{Research questions}

1. RQ: Will there be significant differences between the investigated subjects in muscle hypertrophy at 5-week follow-up?

2. RQ: Will there be significant differences between the subjects studied in the increase in maximum force of the extensors and flexors of the upper limbs at 5-week follow-up?

3. RQ: Will there be significant differences between the study subjects in the reduction of adipose tissue at 5-week follow-up? 


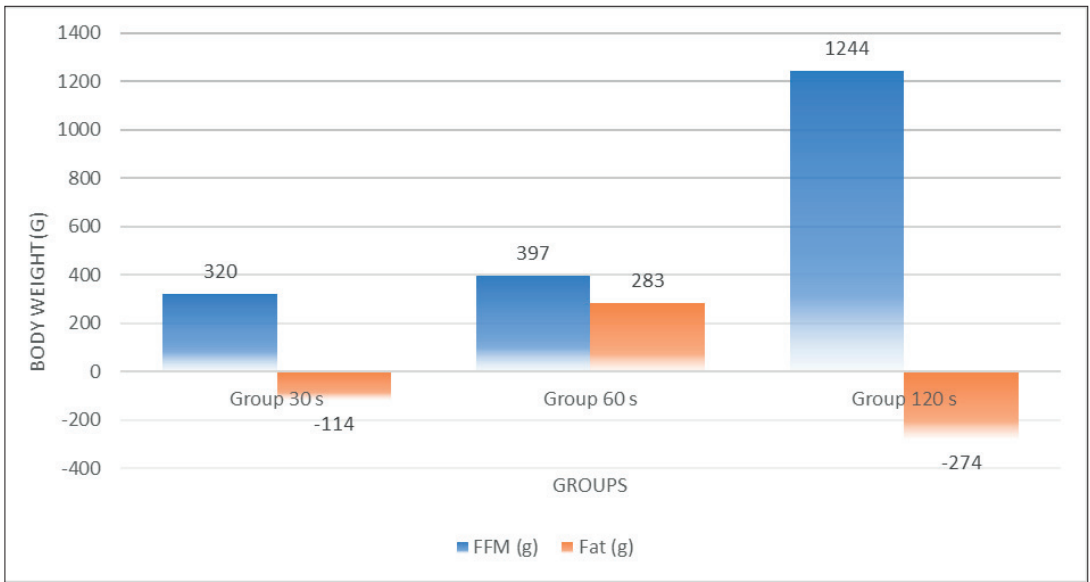

Figure 1. Changes in body composition (g)

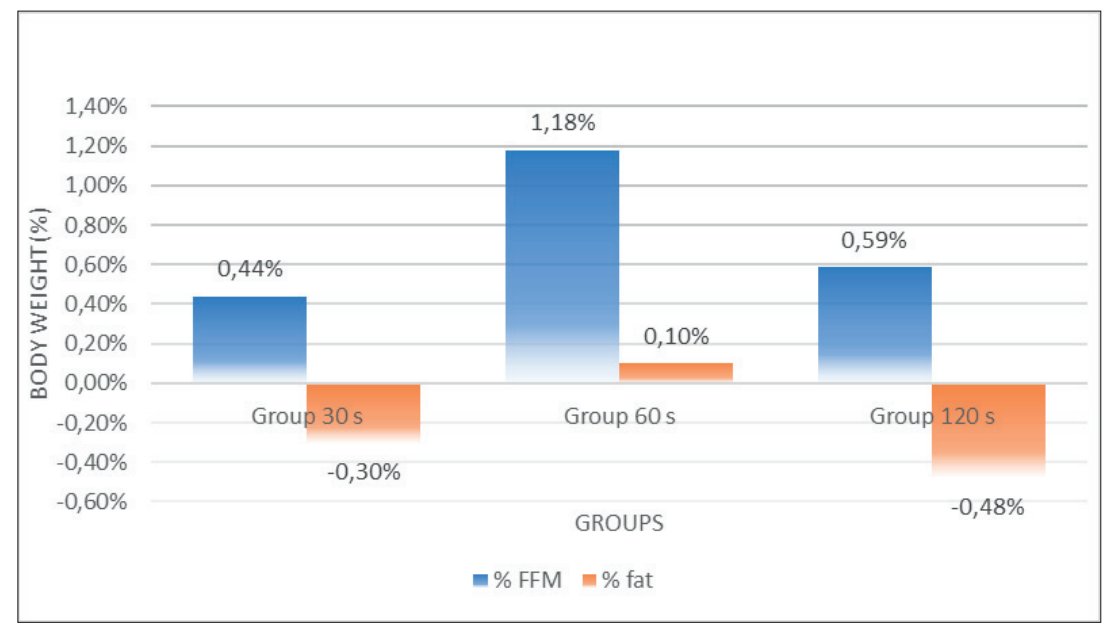

Figure 2. Changes in body composition (\%)

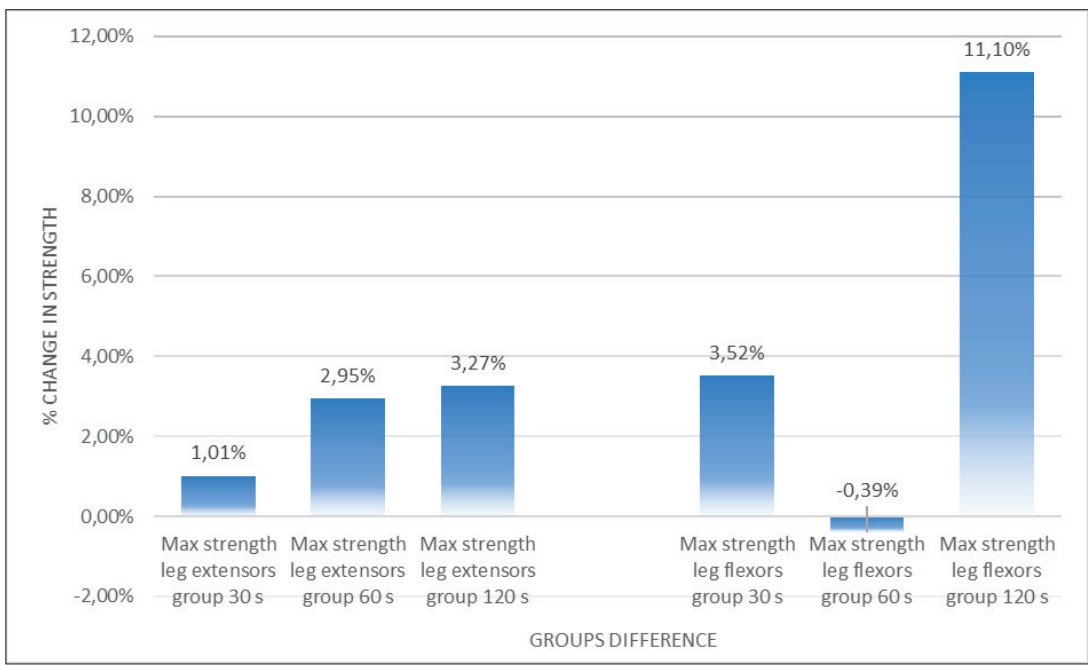

Figure 3. Changes in Lmaximal strength (legs) 


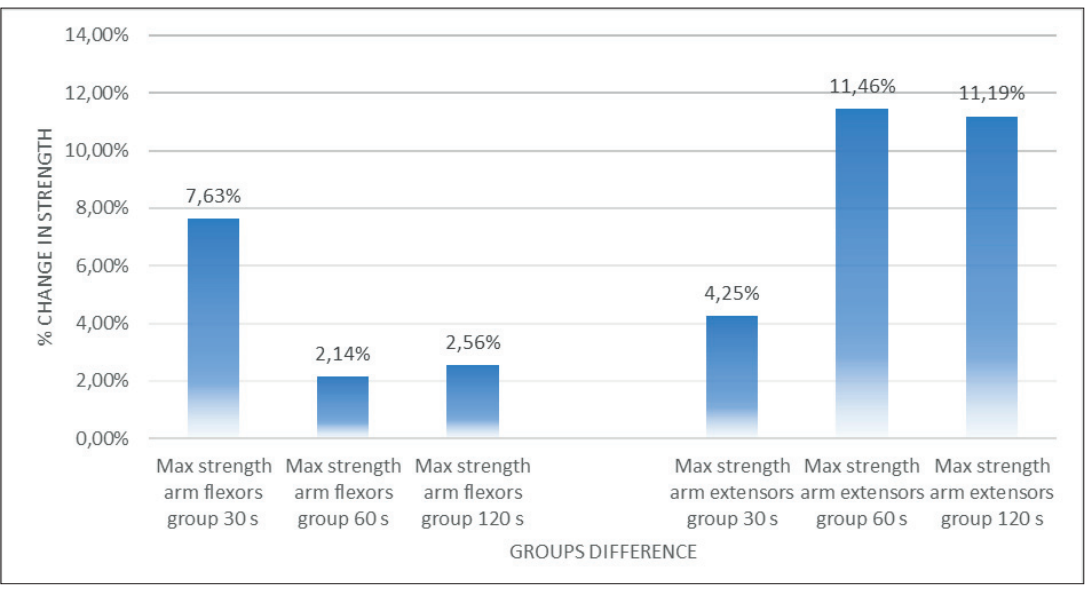

Figure 4. Changes in maximal strength (arms)

Table 1. Changes in body composition

\begin{tabular}{lccrc}
\hline & FFM $(\mathrm{g})$ & Fat $(\mathrm{g})$ & \% fat & $\%$ FFM \\
\hline Group 30 s & 320 & -114 & $-0.30 \%$ & $0.44 \%$ \\
Group 60 s & 397 & 283 & $0.10 \%$ & $1.18 \%$ \\
Group 120 s & 1244 & -274 & $-0.48 \%$ & $0.59 \%$ \\
\hline
\end{tabular}

Table 2. Changes in arm strength

\begin{tabular}{lc}
\hline Group and body part & Change (\%) \\
\hline Max strength of arm flexors group 30 s & $7.63 \%$ \\
Max strength of arm flexors group $60 \mathrm{~s}$ & $2.14 \%$ \\
Max strength of arm flexors group $120 \mathrm{~s}$ & $2.56 \%$ \\
\hline Max strength of arm extensors group 30 s & $4.25 \%$ \\
Max strength of arm extensors group 60 s & $11.46 \%$ \\
Max strength of arm extensors group 120 s & $11.19 \%$ \\
\hline
\end{tabular}

Table 3. Changes in leg strength

\begin{tabular}{lc}
\hline Group and body part & Change (\%) \\
\hline Max strength of leg extensors group 30 s & $1.01 \%$ \\
Max strength of leg extensors group 60 s & $2.95 \%$ \\
Max strength of leg extensors group $120 \mathrm{~s}$ & $3.27 \%$ \\
\hline Max strength of leg flexors group 30 s & $3.52 \%$ \\
Max strength of leg flexors group 60 s & $-0.39 \%$ \\
Max strength of leg flexors group 120 s & $11.10 \%$ \\
\hline
\end{tabular}




\section{RESULTS}

\section{Body composition}

After the 5 week training program we found the following results. At a statistical significance level of 5\%, there was no significant difference in muscle tissue hypertrophy. Group 3 (120 s rest) achieved a four-fold increase in FFM compared to the other two groups in absolute numbers $(1244 \mathrm{~g})$. But the largest percentage increase was achieved in group $2(60 \mathrm{~s})$, with an increase of $1.18 \%$.

In absolute numbers, the highest muscle hypertrophy was seen in group 3 (120 s), which had an average of $1224 \mathrm{~g}$, and which was more than in groups 1 and 2. These groups had total hypertrophy of $320 \mathrm{~g}$ and $397 \mathrm{~g}$ respectively. However, percentages are more relevant for determining the impact of training. Here, the greatest hypertrophy was achieved by group 2 with an increase of $1.18 \%$, followed by group 3 with an increase of $0.59 \%$ and group 1 with an increase of $0.44 \%$ (figure 1 and 2 ).

The highest fat loss occurred in group 3 (120 s), with an average loss of $274 \mathrm{~g}$. Fat loss was also recorded in group 1, the group with the shortest rest time of 30 seconds, and totalled $114 \mathrm{~g}$. The group with a mean rest period of 60 seconds gained $283 \mathrm{~g}$ of body fat.

The highest changes in percentage of body fat were greatest in group 3 with the longest rest time, namely $-0.48 \%$. The remaining two groups demonstrated a decrease of $0.3 \%$ body fat in group 1 , and an increase of $0.1 \%$ of body fat in group 2 .

\section{Muscle strength}

At a statistical significance level of 5\%, we noticed significant differences in the increase in maximum force of the knee joint flexors in only group 3 (11.1\%). Significant differences in muscle strength increase were also observed in groups 2 and 3 in the elbow joint extensors $(11.46 \%$ and $11.19 \%$ respectively) and in the elbow joint flexors only in group $1(7.63 \%)$.

\section{DISCUSSION}

The aim of this study was to determine which of the rest times in strength training has the highest efficiency in relation to the growth of muscle mass and strength. Simultaneously, a further goal was to unify the current ambiguous results of professional studies dealing with the topic of the development of muscle mass in athletes. For higher specificity, we selected experienced athletes from a coordination-aesthetic sport i.e. bodybuilding.

In earlier studies Willardson (2006), Goto (2004), Ratames (2007), Kreamer (1990), Kreamer (1991) and Miranda (2007), the authors agree on the effect of strength training using different rest periods on the development of muscle tissue. Goto (2004) reports short rest intervals (30 s) as being suitable for muscle tissue growth, while Kreamer $(1990,1991)$ recommends the use of rest periods of 60-90 seconds and Miranda (2007) suggests the possibility of using long pauses (above $120 \mathrm{~s}$ ) for adequate muscle hypertrophy. The authors do not agree on the ideal length of rest period or the development of muscle tissue. However, there is agreement that there should not be an ideal rest period between work series for the development of maximum strength.

In the study, we have demonstrated the effect of strength training on body composition, both on the amount of muscle tissue and body fat. These were measured using a DEXA bone densitometer. We have also demonstrated the effect of strength training on muscle strength and the effect of maximum force measured on an angular isokinetic dynamometer. 
The highest relative change in muscle mass was recorded in group 2. This does not confirm the previously highlighted assumption that longer pauses also mean higher gains in muscle tissue, as the increase in muscle mass of $1.18 \%$ was not significant. However, in regard to the experience of the participants (who had more than 4 years of systematic training) and the length of the intervention, this result can be considered interesting and usable in training practice. Experienced athletes do not see such a rapid increase in muscle mass as beginners, so we can consider this percentage increase to be beneficial. Changes in body fat composition did not show a direct dependence on the rest break used in the training plan.

Changes in strength dispositions were not a direct goal of the training plan, which was conceived as a "hypertrophic training plan". Even so, there were significant changes in the increase in strength, although unevenly across the groups (figure 3, figure 4). This can be attributed to the inappropriate prioritization of some muscle groups in traditional bodybuilding training plans and the correction of muscle imbalances based on the training plan used during the experiment.

The answers to the research questions are based on following sentences. We did not find significant differences between the groups in muscle hypertrophy (RQ1). However, significant differences were found between the groups in the increase in maximum strength of the extensors and flexors of the upper and lower limbs. (RQ2) There were no significant differences between the groups in the reduction of adipose tissue (RQ 3) found in the study.

\section{PRACTICAL APPLICATION (CONCLUSION)}

The practical application of the findings will help us to establish more effective training programs that are targeted towards the appropriate rest time needed for muscle hypertrophy. It will also allow us to shorten the required training unit. This information may find practical application in bodybuilding training and in the creation of fitness training plans that are aimed at increasing muscle mass and strength.

\section{Acknowledgement}

This manuscript is original and not previously published in any form including on preprint servers.

\section{References}

1. Schiaffino, S., Hanzlíková, V. On the mechanism of compensatory hypertrophy in skeletal muscles. Experientia 26, $152-153,1970$

2. Schoenfeld, BJ. The mechanisms of muscle hypertrophy and their application to resistance training. J Strength Cond Res24 (10): 2857-2872, 2010

3. Maughan, RJ, Watson, JS, and Weir, J. Strength and cross-sectional area of human skeletal muscle. J Physiology 338 : 37-49, 1983.

4. Evans, WJ. Effects of exercise on senescent muscle. Clin Orthopaed, Rel Res 403(Suppl.): S211-S220, 2002.

5. Baltzopoulos V, Brodie DA. Isokinetic dynamometry. Applications and limitations. Sports Med. 1989

6. Jones, DA and Rutherford, OM. Human muscle strength training: The effects of three different regimens and the nature of the resultant changes. J Physiol 391: 1-11, 1987.

7. Schwarzenegger, A, Dobbins, B. The new encyclopedia of modern bodybuilding. New York: Simon \& Schuster, 1999

8. Valkeinen $\mathrm{H}$, Häkkinen $\mathrm{K}$, Pakarinen A, et al. Muscle hypertrophy, strength development, and serum hormones during strength training in elderly women with fibromyalgia. Scand J Rheumatol.34(4): 309-314, 2005

9. Ahtiainen, JP, Pakarinen, A, Alen, M, Kraemer, WJ, and Häkkinen, K. Muscle hypertrophy, hormonal adaptations and strength development during strength training in strength-trained and untrained men. Eur J Appl Physiol 89: 555-563, 2003.

10. Porter, Michelle \& Vandervoort, A.A. \& Lexell, Jan. (1995). Aging of human muscle: structure, function and adaptability. Scandinavian journal of medicine \& science in sports. 5. 129-42, 1995 
STUDIA SPORTIVA $2021 / 1$

11. McCartney N, Hicks, A, Martin, J, Webber, C. A Longitudinal Trial of Weight Training in the Elderly: Continued Improvements in Year 2, The Journals of Gerontology: Series A, Volume 51A, Issue 6, p. B425-B433, 1996

12. Häkkinen, $\mathrm{K}$, Pakarinen, $\mathrm{A}$, Alen, $\mathrm{M}$, Kauhanen, $\mathrm{H}$, and Komi, PV. Neuromuscular and hormonal adaptations in athletes to strength training in two years. J Appl Physiol 65: 2406-2412, 1988.

13. Häkkinen, K, Pakarinen, A, Kraemer, WJ, Newton, RU, and Alen, M. Basal concentrations and acute responses of serum hormones and strength development during heavy resistance training in middle-aged and elderly men and women. J Gerontol. Ser A, Biol Sci Med Sci 55: B95-B105, 2000.

14. Häkkinen, K, Newton, R, Gordon, SE, McCormick, M, etc. Changes in Muscle Morphology, Electromyographic Activity, and Force Production Characteristics During Progressive Strength Training in Young and Older Men, The Journals of Gerontology: Series A, Volume 53A, Issue 6, Pages B415-B423, 1998

15. Michels, G and Hoppe, UC. Rapid actions of androgens. Fron Neuroendocrin 29: 182-198, 2008.

16. Grgic J, Lazinica B, Mikulic P, Krieger JW, Schoenfeld BJ. The effects of short versus long inter-set rest intervals in resistance training on measures of muscle hypertrophy: A systematic review. Eur J Sport Sci.;17(8): 983-993, 2017

17. Prestes J, A Tibana R, de Araujo Sousa E, et al. Strength and Muscular Adaptations After 6 Weeks of Rest-Pause vs. Traditional Multiple-Sets Resistance Training in Trained Subjects. J Strength Cond Res.;33 Suppl 1:S113-S121, 2019

18. Kraemer, WJ, Hakkinen, K, Newton, RU, Nindl, BC, Volek, JS, McCormick, M, Gotshalk, LA,Gordon, SE, Fleck, SJ, Campbell,WW, Putukian, M, and Evans, WJ. Effects of heavy-resistance training on hormonal response patterns in younger vs. older men. J Appl Physiol 87: 982-992, 1999.

19. Häkkinen K, Häkkinen A. Neuromuscular adaptations during intensive strength training in middle-aged and elderly males and females. Electromyogr Clin Neurophysiol.;35(3):137-147, 1995

20. Kraemer, WJ, Marchitelli, L, Gordon, SE, Harman, E, Dziados, JE, Mello, R, Frykman, P, McCurry, D, and Fleck, SJ. Hormonal and growth factor responses to heavy resistance exercise protocols. J Appl Physiol 69: 1442-1450, 1990.

21. Kraemer, WJ, Gordon, SE, Fleck, SJ, Marchitelli, LJ, Mello, R, Dziados, JE, Friedl, K, Harman, E, Maresh, C, and Fry, AC. Endogenous anabolic hormonal and growth factor responses to heavy resistance exercise in males and females. Int $J$ Sport Med 12: 228-235, 1991

22. Willardson, JM. A brief review: Factors affecting the length of the rest interval between resistance exercise sets. $J$ Strength Cond Res 20: 978-984, 2006.

23. Kraemer, WJ, Noble, BJ, Clark, MJ, and Culver, BW. Physiologic responses to heavy-resistance exercise with very short rest periods. Int J Sport Med 8: 247-252, 1987.

24. Goto, K, Ishii, N, Kizuka, T, and Takamatsu, K. The impact of metabolic stress on hormonal responses and muscular adaptations. Med Sci Sport Exerc 37: 955-963, 2005.

25. Miranda, H, Fleck, SJ, Simão, R, Barreto, AC, Dantas, EH, and Novaes, J. Effect of two different rest period lengths on the number of repetitions performed during resistance training. J Strength Cond Res 21: 1032-1036, 2007

26. Ratamess, NA, Falvo, MJ, Mangine, GT, Hoffman, JR, Faigenbaum, AD, and Kang, J. The effect of rest interval length on metabolic responses to the bench press exercise. Eur J Appl Physiol 100: 1-17, 2007.

27. Moritani, T, Neuromuscular adaptations during the acquisition of muscle strength, power and motor tasks, Journal of Biomechanics, Volume 26, Supplement 1: 95-107, 1993 


\section{SUPPLEMENT}

\section{Supplement 1. - training plan}

- Training with $80 \%$ of 1RM (1 repetition maximum)

- In the third week you go to $85 \%$ of $1 \mathrm{RM}$

- TUT (time under tension) = 3-0-1-0

- Rest period: 30,60 or 120 seconds.

- Cardiovascular training is recommended as active regeneration after strength training

\section{Weekly Training Plan}

- Mon - training 1

- Tue - training 2 - cardio activity $10 \mathrm{~min}$

- Wed - free day

- Thu - training 3

- Fri - training 4

- Sat - training 5 - cardio activity $10 \mathrm{~min}$

- Sun - free day

- Repeat the cycle for subsequent weeks

\section{Training Components}

1) Warm up with 5-10 min of cardio activity

2) Joint mobilisation

3) Dynamic stretching and core activation

4) Main training program

Training 1

\begin{tabular}{lcc}
\hline Exercise & Series & Repetition/weight (\% 1RM) \\
\hline Dumbbell bench press & $4^{*}$ & $7-8(80 \%)$ \\
Incline benchpress & $3^{*}$ & $7-8(80 \%)$ \\
Incline fly press & 3 & $7-8(80 \%)$ \\
Pec deck & 3 & $7-8(80 \%)$ \\
Cable fly & 3 & $7-8(80 \%)$ \\
Dumbbell curl & 4 & $7-8(80 \%)$ \\
Hammer curl & 4 & $7-8(80 \%)$ \\
\hline
\end{tabular}

* before the main series, a series with $20 \%$ and $40 \% 1 \mathrm{RM}$ is completed

\section{Training 2}

\begin{tabular}{lcc}
\hline Exercise & Series & Repetition/weight (\% 1RM) \\
\hline Dumbbell shoulder press & $4^{*}$ & $7-8(80 \%)$ \\
Machine shoulder press & 3 & $7-8(80 \%)$ \\
Lateral raise & 3 & $7-8(80 \%)$ \\
Frontal raise & 3 & $7-8(80 \%)$ \\
Rope tricep pushdowns & 4 & $7-8(80 \%)$ \\
Overhead tricep extension & 4 & $7-8(80 \%)$ \\
\hline
\end{tabular}

* before the main series, a series with $20 \%$ and $40 \% 1 \mathrm{RM}$ is completed 
STUDIA SPORTIVA $2021 / 1$

Training 3

\begin{tabular}{lcc}
\hline Exercise & Series & Repetiton/weight (\% 1RM) \\
\hline Back squat & $4^{*}$ & $7-8(80 \%)$ \\
Leg press & $3^{*}$ & $7-8(80 \%)$ \\
Quadricep extensions & 3 & $7-8(80 \%)$ \\
Prone leg curl & 3 & $7-8(80 \%)$ \\
Romanian deadlift & 3 & $7-8(80 \%)$ \\
Standing calf raises & 3 & $6-12(80 \%)$ \\
\hline
\end{tabular}

* before the main series, a series with $20 \%$ and $40 \% 1 \mathrm{RM}$ is completed

Training 4

\begin{tabular}{lcc}
\hline Exercise & Series & Repetiton/weight (\% 1RM) \\
\hline Lat pulldown & $4^{*}$ & $7-8(80 \%)$ \\
Pull ups & 3 & $7-8(80 \%)$ \\
Bent-over barbell rows & 3 & $7-8(80 \%)$ \\
Seated cable rows & 3 & $7-8(80 \%)$ \\
Cable pullover & 4 & $7-8(80 \%)$ \\
Hyperextension & 3 & 15 \\
\hline
\end{tabular}

* before the main series, a series with $20 \%$ and $40 \% 1 \mathrm{RM}$ is completed

Training 5

\begin{tabular}{lcc}
\hline Exercise & Series & Repetiton/weight (\% 1RM) \\
\hline Bicep curl & $4^{*}$ & $7-8(80 \%)$ \\
Preacher curl & 3 & $7-8(80 \%)$ \\
Tricep benchpress & $4^{*}$ & $7-8(80 \%)$ \\
Tricep dips & 3 & $7-8(80 \%)$ \\
Rope tricep pushdowns & 4 & $7-8(80 \%)$ \\
Crunches & 3 & $7-8(80 \%)$ \\
Sitting twists & 3 & $7-8(80 \%)$ \\
\hline
\end{tabular}

* before the main series, a series with $20 \%$ and $40 \% 1 \mathrm{RM}$ is completed 